\title{
An Effective SPC Approach to Monitoring Semiconductor Quality Data with Multiple Variation Sources
}

\author{
Argon Chen, Ruey-Shan Guo, and Pei-Chen Yeh \\ Graduate Institute of Industrial Engineering \\ National Taiwan University \\ 1, Roosevelt Rd. Sec. 1, Taipei, Taiwan 106
}

Fax: 886-2-23635856 Email: achen@,ccms.ntu.edu.tw

\begin{abstract}
In semiconductor manufacturing, postprocess metrology and statistical monitoring of quality data are critical to ensure a high yield and good quality products. Effective SPC charting can detect process excursions and provide early warning on possible process faults. Therefore, it is important to design SPC charts such that process shifts, small or large, can be detected early and accurately while the number of false alarms can be cut down to an acceptable level.
\end{abstract}

\section{Introduction}

The problem most often encountered by process engineers while doing SPC charting in semiconductor fabrication is the difficulty to group the data and compute the sample statistic to be charted. Due to the high cost of test wafers and metrology, only few sample wafers are taken from a lot or a process run. Sample readings are then taken from the sample wafers. For example, after oxidation five thickness readings will be taken from each of three sample wafers (Fig. 1), which in turn are taken from a processing batch.

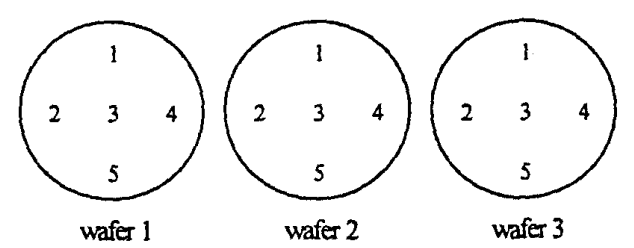

Fig. 1 Thickness sample readings

With these sample thickness data, the most often seen practice is to group 5 sample readings from a wafer to compute an average. The computed wafer averages are then plotted on the popular $\bar{X}$ control chart. This is not the only possibility of grouping the data. We could be more concerned about the batch-to-batch variation and decide to group all 15 readings together for computing batch averages and plotting a batch-to-batch control chart. We could be also concerned about the site-to-site variation and decide to group the readings from the same site (e.g. site 1) together. 5 sit-to-site (one for each) control charts can be then constructed to detect problems that only reveal themselves in the site-to-site relationships. There of course exist other possibilities. It all depends on what variation sources you are concerned with.

In this paper, we first present an analysis of variance (ANOVA) that decomposes and discerns significant variation sources. Such analyses provide insights on what sources of variation should be watched more closely. Table 1 shows an ANOVA for the oxidation thickness.

Table 1: ANOVA for Oxidation Thickness

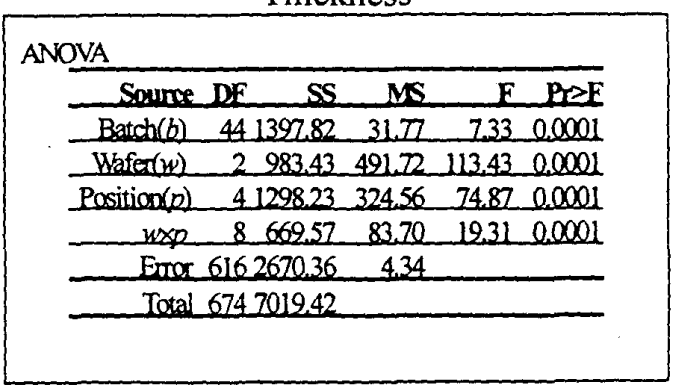

From the analysis of variance, it is found that all the main variation sources (batch-to- 
batch, wafer-to-wafer, and site-to-site) are quite significant. In addition, the interaction between wafers and positions is also discernible. Therefore, control charts should be constructed to monitor these variation sources.

\section{Control Charting}

Fig.2 shows the four batch-to-batch control chart that are most often seen in practice. One is an "overall" batch-to-batch chart in that each data point is the average of all 15 readings from the same batch. The other three $\vec{X}-R$ charts are "zone" batch-tobatch charts. Since wafers 1, 2, 3 are taken, respectively, from top; center, and bottom zones of a vertical oxidation furnace, each data point on a "zone" batch-to-batch chart is an average or range of the 5 readings from each wafer. For example, in the "top-zone" control chart each data point represents the thickness average of waferl from one batch.
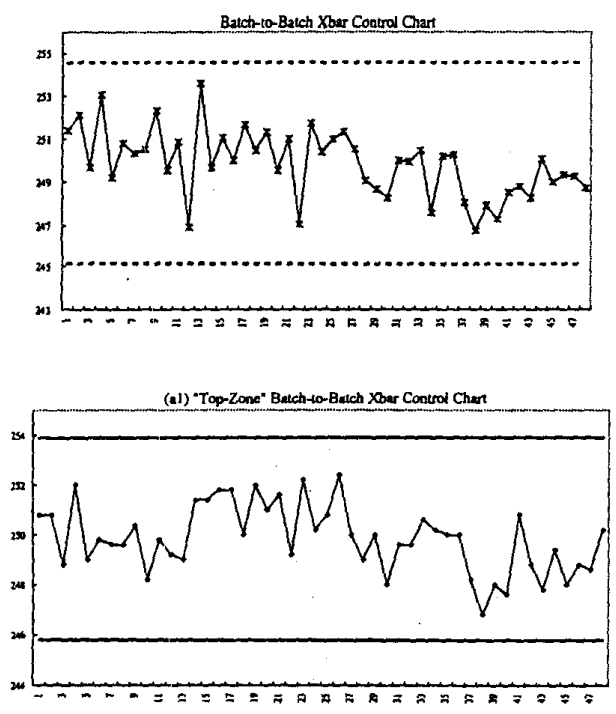
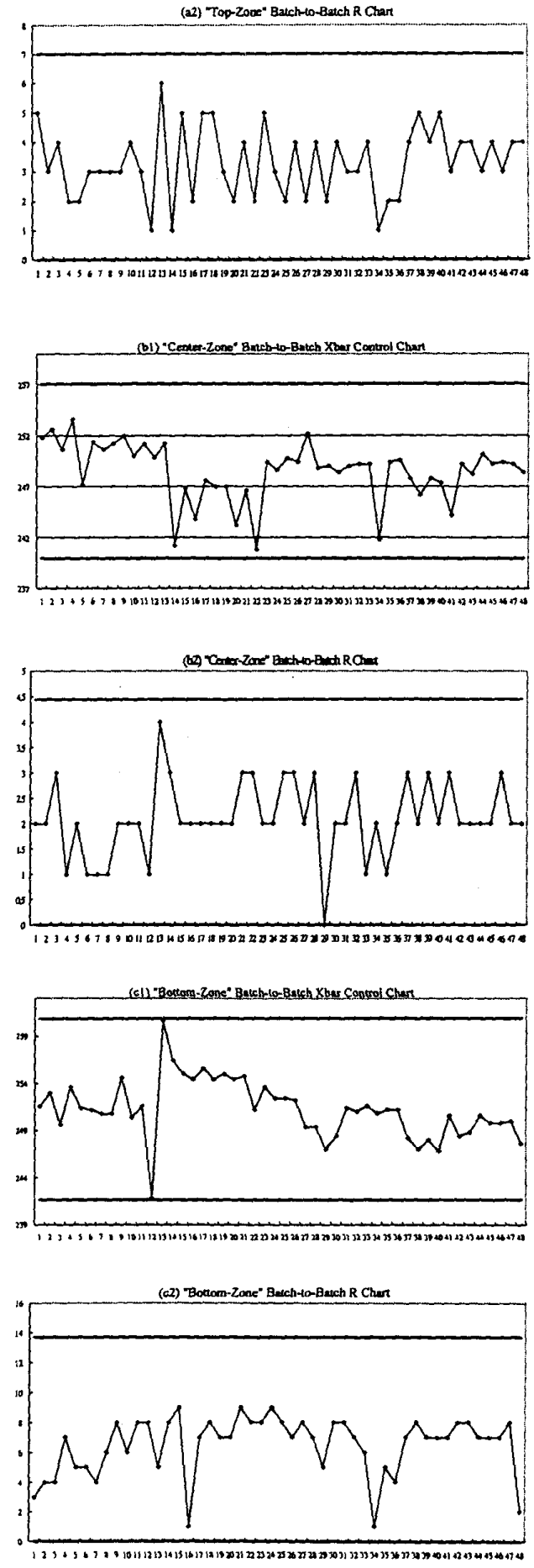

Fig. 2 Four most used batch-to-batch charts 
Tocapture all variation sources, it is not suffofeient to only rearrange and regroup the sample data. The difficulty is always that there exist too many control charts and thus too many false alarms and there simply lack effective control charts to provide accurate detection of problems. In this paper, we propose an integrated, effective SPC approach that can detect excursions from all sorts of variation sources. The same thickness data from an oxidation process is used to illustrate the effectiveness of our proposed approach. From Fig. 2, the process seems running $\mathrm{OK}$ since all data points are within the control limits. What cannot be seen in Fig. 2 are a lot of hidden variation sources, that hid behind the sample averages [1].

In order to capture and describe all possible variation sources, we here propose the following model:

$X_{i j k}=\mu+b_{i}+w_{j(i)}+p_{j k(i)}$,

where each thickness reading $\left(X_{i j k}\right)$ taken

from batch $i$, wafer $j$ and position (site) $k$ can

be explained by four components:

$\mu$ : overall mean

$b_{i}$ : batch effect $\left(\sim \mathrm{N}\left(0, \sigma_{b}^{2}\right)\right)$

$w_{j(i)}$ : wafer effect $\left(\left[w_{j(i)}\right]_{m \times 1} \sim \mathrm{N}\left(\boldsymbol{\mu}_{w}, \boldsymbol{\Sigma}_{w}\right)\right.$

$j=1, \ldots, m)$ and

$p_{j k(i)}$ : position effect $\left(\left[p_{j k(i)}\right]_{n \times 1} \sim \mathrm{N}\left(\boldsymbol{\mu}_{j}, \boldsymbol{\Sigma}_{j}\right)\right.$,

$k=1 . . n)$.

Here, we also assume $b_{i} \cdot p_{j k(i)} \cdot w_{j(i)}$ are dependent of one another. Without loss of generality,

$$
\sum_{j=1}^{m} \mu_{w_{j}}=0 \quad, \quad \sum_{k=1}^{n} \mu_{j k}=0, j=1 . . m .
$$

In model (1), $b_{i}$ represents the deviation from the overall mean $(\mu)$ in the $i$ th batch caused by batch-to-batch variation. The size of the batch-to-batch deviation is assumed to follow a normal distribution. $\boldsymbol{w}_{(i)}$ represents the variation among wafers from the same batch and is an $m \times 1$ vector following a multivariate normal distribution. Similarly, $\mathbf{p}_{j(i)}$ represents variations among positions on wafer $j$ of batch $i$ and is an $n \times 1$ vector following a multivariate normal distribution. In the case of Fig. 1: $m=3, n=5$ and

$$
\begin{aligned}
& w_{(i)}= \\
& {\left[\begin{array}{l}
w_{1(i)} \\
w_{2(i)} \\
w_{3(i)}
\end{array}\right] \sim N\left(\mu_{w}, \Sigma_{w}\right)=N\left(\left[\begin{array}{l}
\mu_{w_{1}} \\
\mu_{w_{2}} \\
\mu_{w_{3}}
\end{array}\right],\left[\begin{array}{lll}
\sigma_{w_{1}}^{2} & \sigma_{w_{1}, w_{2}} & \sigma_{w_{1}, w_{3}} \\
\sigma_{w_{1}, w_{2}} & \sigma_{w_{2}}^{2} & \sigma_{w_{2}, w_{3}} \\
\sigma_{w_{1}, w_{3}} & \sigma_{w_{2}, w_{3}} & \sigma_{w_{3}}^{2}
\end{array}\right]\right)} \\
& p_{\mathrm{j}(i)}=\left[\begin{array}{l}
p_{j 1(i)} \\
p_{j 2(i)} \\
p_{j 3(i)} \\
p_{j 4(i)} \\
p_{j 5(i)}
\end{array}\right] \sim N\left(\mu_{j}, \Sigma_{j}\right)=
\end{aligned}
$$

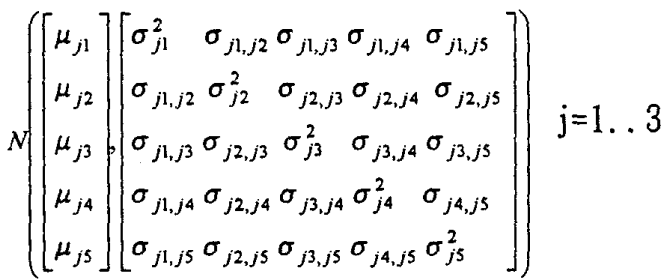

4 multivariate $\mathrm{T}^{2}$ control charts [2] are then proposed to monitor these difference variation components (to be described in detailed in the full paper). These four control charts are:

1.position (site) effect chart for wafer 1 (top zone)

2. position effect chart for wafer 2 (center zone)

3.position effect chart for wafer 3 (bottom zone) and

4.wafer effect chart

Fig. 3 shows these four $T^{2}$ charts. It is seen that the control chart can effectively detect the problem batches 12,13 , and 29 and identify their variation sources: wafer variation plus bottom-zone position variation for batch 12 , wafer variation plus center-zone position variation for batch 13 and bottom-zone position variation for batch 29. In the mean time, it can be also observed that the in-control batches are all very well kept within the control limits. This 
illustrates the control charts' robustness against false alarms.

In conclusion, the proposed SPC approach is shown to be very effective in detecting process excursions with multiple variation sources and is also robust by keeping the in-control data points well within the control limits. This technique is especially useful for semiconductor manufacturing. Post-process sample data, such as thickness, $\mathrm{CD}$ and alignment data, can be effectively monitored. The technique can also provide engineers useful information on the source of excursion.

\section{References:}

1. Argon Chen, R. S. Guo and C. L. Lee, "Statistical monitoring of thickness data using bivariate runs rules," The $7^{\text {th }}$ ISSM, 452-455, Tokyo, 1998.

2.F. b. Alt, "Multivariate Quality Control" in Encyclopedia of Statistical Sciences 6. John Wiley \& Sons, New York, NY, 1985.
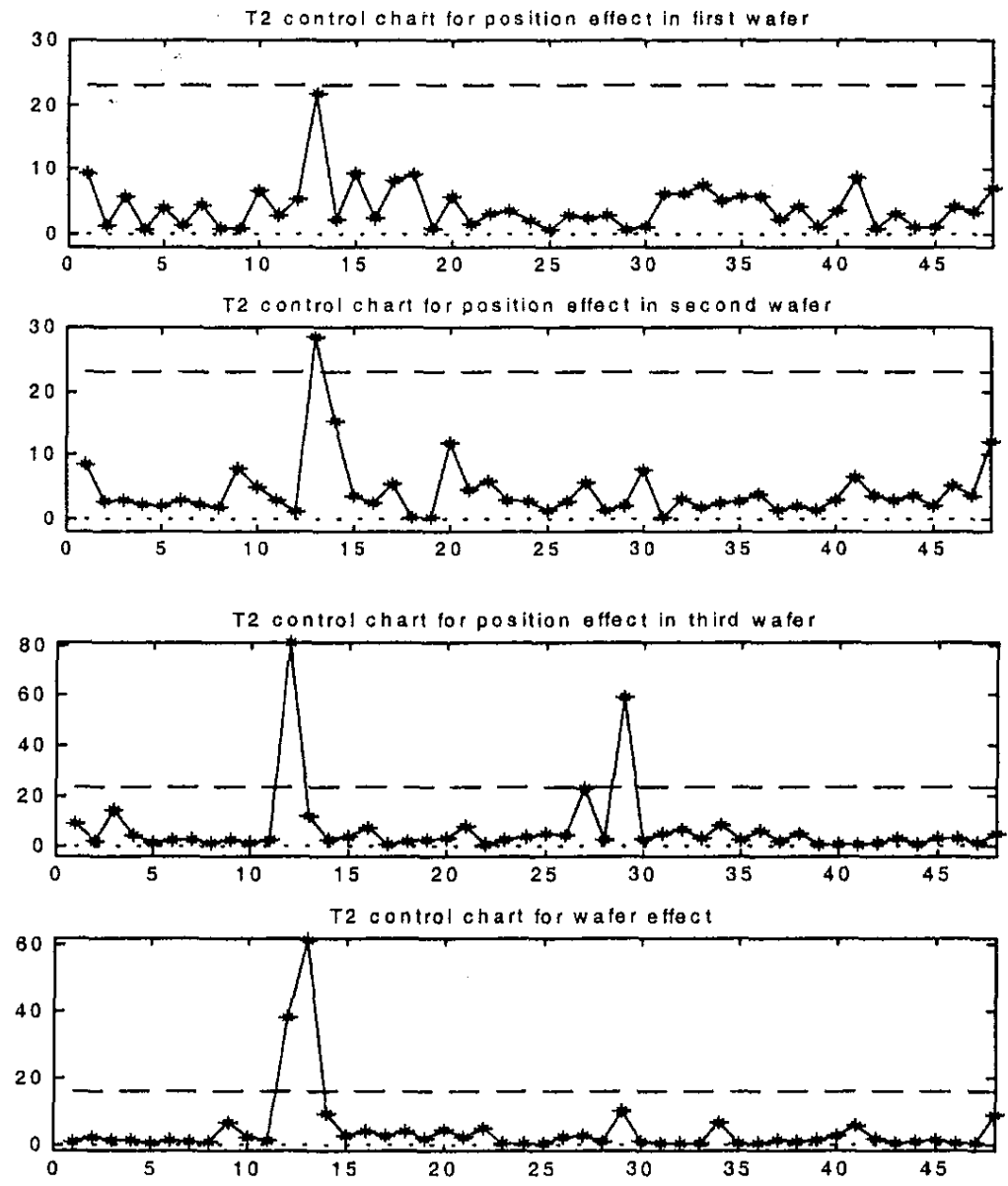

Fig. $3 \mathrm{~T}^{2}$ control charts for multiple variation sources 